\title{
A Comparative Study of Suicide Rates among 10-19-Year-Olds in 29 OECD Countries
}

\author{
Beop-Rae Roh', Eun Hee Jung ${ }^{2}$, and Hyun Ju Hong ${ }^{1,3 凶}$ \\ ${ }^{1}$ Hallym University Suicide and School Mental Health Institute, Anyang, Republic of Korea \\ ${ }^{2}$ Mental Health and Communal Society Research Center, Kwangwoon University, Seoul, Republic of Korea \\ ${ }^{3}$ Department of Psychiatry, Hallym University Sacred Heart Hospital, Anyang, Republic of Korea
}

\begin{abstract}
Objective This study had two main objectives: to compare current suicide rates in OECD countries among 10-19-year-olds and to identify patterns of suicide rates based on age, gender and time. Furthermore we investigated the main dimensions that contributed to the variation in child and adolescent suicide rates across countries.
\end{abstract}

Methods We combined the WHO mortality data and the population data released by OECD to calculate the suicide rates in 29 OECD countries. A self-organizing map (SOM), k-means clustering analysis, and multi-dimensional scaling were used to classify countries based on similarities in suicide rate structure and to identify the important dimensions accounting for differences among groups.

Results We identified significant differences in suicide rates depending on age, sex, country, and time period. Late adolescence and male gender were universal risk factors for suicide, and we observed a general trend of declining suicide rates in OECD countries. The SOM analysis yielded eight types of countries. Most countries showed gender gaps in suicide rates of similar magnitudes; however, there were outliers in which the gender gap was particularly large or small.

Conclusion Significant variation exists with respect to suicide rates and their associated gender gaps in OECD countries.

Psychiatry Investig 2018;15(4):376-383

Key Words Suicide, Child, Adolescent, OECD, Comparative study.

\section{INTRODUCTION}

Globally, suicide is the second leading cause of mortality among young people. ${ }^{1}$ Suicide is a complex phenomenon involving individual, familial, cultural, and social factors, and varying suicide rates have been reported in different countries and at different times. Although adolescent suicide is relatively infrequent compared with that in older age groups, it is a major public health concern in many countries. ${ }^{2}$ Studies regarding the characteristics that underlie differences in suicide rates across various countries have the potential to yield meaningful insights for interventions and related policies.

Suicide rates differ by age and gender. ${ }^{3-12}$ The absolute num-

\section{Received: June 8, 2017 Accepted: August 2, 2017}

$\triangle$ Correspondence: Hyun Ju Hong, MD, PhD

Department of Psychiatry, Hallym University Sacred Heart Hospital, Hallym University College of Medicine, 22 Gwanpyeong-ro 170beon-gil, Dongan-gu, Anyang 14068, Republic of Korea

Tel: +82-31-380-3750, Fax: +82-31-381-3753, E-mail: honghj88@gmail.com (c) This is an Open Access article distributed under the terms of the Creative Commons Attribution Non-Commercial License (http://creativecommons.org/licenses/bync/4.0) which permits unrestricted non-commercial use, distribution, and reproduction in any medium, provided the original work is properly cited. ber of completed suicides among male adolescents is higher than that among female adolescents, ${ }^{13}$ whereas suicidal ideation and attempts are much more frequent among female adolescents; this is frequently referred to as the gender paradox of suicidal behavior., ${ }^{4,14,15}$ Universally, completed suicide before puberty is rare, ${ }^{1,16}$ with the incidence of suicide increasing markedly in the late teens and continuing to rise. ${ }^{17,18}$ This is reflected in a global increase from 0.6 completed suicides per 100,000 individuals among youth aged 14 or younger ${ }^{19}$ to a mean worldwide suicide rate of 6.9/100,000 among teenagers between the ages of 15 and 19 years. ${ }^{20}$ However, the magnitude of the effects of gender and age on suicide rates varies considerably across countries. ${ }^{20}$ In some Asian countries, including China and India, youth suicide rates for females exceed those for males, ${ }^{21-23}$ and in Western countries, suicide rates for females aged 14 or younger have been reported to be increasing..$^{24}$ In short, although similar overall patterns with regard to the effects of age and gender on suicide rates are observed in most countries, the magnitude of differences due to age and gender varies significantly by region.

Characterizing the patterns underlying suicide rates becomes 
even more complex if the effects of time are considered in addition to gender and age. Suicide rates were reported to rise in the 1980s and $1990 \mathrm{~s},{ }^{2,25}$ whereas more recently available data from the $\mathrm{WHO}$ indicate that rates gradually decreased between 2000 and 2009. ${ }^{20}$ Between 2000 and 2009, the teenage suicide rate decreased in North America, whereas it increased in Central and South America in both the 14-and-under and 15-andover age groups. ${ }^{24}$ East Asian countries, such as Japan and South Korea, are characterized by an increasing trend in teenage suicides, with a narrowing gender gap. Southeastern and southern European regions exhibit the lowest overall levels of teenage suicide, with the exception of Australia and New Zealand. Despite the fact that suicide rates have decreased in Eastern Europe, the countries in this region, including Russia and countries formerly belonging to the Soviet Union, continue to have the highest suicide rates. Finally, adolescent suicide rates have decreased slightly in northern Europe, but have remained, on average, fairly stable. ${ }^{20,24,26}$

There has been little research comparing teenage suicide rates across countries based on recent data that simultaneously considered gender, age, and time. Most research has been conducted using data from a single country, ${ }^{12,27-31}$ with some comparative studies describing differences in suicide rates within broader categories beyond the country level. ${ }^{24,26}$

In the present study, we used recent WHO mortality data and OECD population data to compare suicide rates among 10-19-year-olds across 29 OECD countries and to explore the effects of gender, age, and time. We then evaluated the variation in suicide rates across countries and identified patterns of suicide rates based on age and gender. Finally, we investigated the main dimensions that contributed to the variation in child and adolescent suicide rates across countries.

\section{METHODS}

\section{Data}

We calculated the suicide rate for each country by combining the raw WHO mortality data and the OECD population data of 29 member countries for which the latest mortality and population data were available in 2014. We excluded 5 member countries reporting no data on suicide rate or reporting suicide data of less than 5 years. Due to issues related to the accessibility of data, there are some cross-country differences with respect to specific time periods covered, but we obtained data for each country for approximately the period from the mid1990s to about 2012. The WHO mortality data include the number of deaths and their causes based on the International Classification of Diseases (ICD). Deaths classified as "death from intentional self-injury" were deemed to represent suicide, which corresponds to the ICD codes X60-X84. OECD popu- lation data are estimated for 5-year age groups and sex. We combined the WHO mortality data and the OECD population data to determine suicide rates based on the incidence of suicide per 100,000 individuals for each country and year. We calculated suicide rates by gender separately in adolescents aged 10-14 and 15-19. The protocol of this study was reviewed by the Review Board of Hallym University Sacred Heart Hospital (IRB No. 2016-I049).

\section{Statistical analysis}

First, we assessed the overall adolescent suicide rate by gender and age for each country by averaging suicide rates across two separate periods of time. Completed suicide is a rare event in the case of children and adolescents, and annual fluctuations are commonly observed. Hence, even small fluctuations in the number of suicides may significantly affect a country's suicide rate in any given year. To take into account this annual variation in adolescent suicide rates, we calculated the average suicide rates for the period prior to and including 2005 and for the period after 2005. Thus, for each country, we observed suicide rates within two separate time intervals, with each time interval analyzed separately. We set 2005 as the boundary between the two time periods because this year represents the approximate midpoint of the average period for which data were available. Furthermore, we examined differences in suicide rates by age and gender and identified trends occurring in OECD countries between the mid-1990s and the early 2010s.

Next, to examine the gender and age structure of suicide rates across countries, we conducted a classification analysis using a self-organizing map (SOM). SOM is a technique used to identify distances among cases and to visualize the results in the form of a map by projecting the high-dimensional data structure to a flat grid. ${ }^{32,33} \mathrm{SOM}$, also known as a Kohonen Map, is one of the best-known clustering methods for unobserved features. ${ }^{34}$ Cluster structures can be identified as a result of the SOM analysis. Furthermore, in addition to performing clustering, the SOM analysis provides a topological organization of the resulting clusters. ${ }^{33}$ We applied SOM analysis to our suicide data set to reveal cluster structures and relationships related to age and sex, while preserving essential information in our data set to allow for readily understandable comparisons.

Three steps related to classification and analysis were followed in this study. The first step was to categorize cases with similar patterns of youth suicide rates to take advantage of the SOM. The second step involved conducting a k-means clustering analysis on the results of the SOM and determine the membership of each case. We compared the mean suicide rates by age and gender for each group as classified by k-means clustering analysis and examined their features. The third step involved conducting multidimensional scaling (MDS) on the re- 
sults of the previous classification. MDS is an exploratory method used to identify the key dimensions underlying differences among groups. ${ }^{32}$ In this step, our aim was to determine which dimensions were important for understanding differences in the patterns of child and adolescent suicide rates across countries.

\section{RESULTS}

\section{Comparing suicide rates by countries}

Table 1 summarizes the child and adolescent suicide rates in the 29 countries included in this study. Countries are arranged in descending order based on their overall average suicide rate, and the suicide rates for each country are broken down into eight categories. The second column of Table 1 shows the time period of data included in the analysis.

We observed differences in suicide rates based on age, sex, country, and time period. The suicide rates in the 15-19-year age group were higher than those in the 10-14-year age group. Age had a stronger effect on suicide rates for males than it does for females. In males, the overall suicide rate in the 15-19-year age group was 7.93 times higher than that in the 10-14-year

Table 1. Child and adolescent suicide rates of 29 countries (per 100,000)

\begin{tabular}{|c|c|c|c|c|c|c|c|c|c|c|}
\hline \multirow{3}{*}{ Country } & \multirow{3}{*}{ Period } & \multicolumn{4}{|c|}{ Age: $10-14$} & \multicolumn{4}{|c|}{ Age: $15-19$} & \multirow{3}{*}{$\begin{array}{l}\text { Total } \\
\text { mean }\end{array}$} \\
\hline & & \multicolumn{2}{|c|}{ Male } & \multicolumn{2}{|c|}{ Female } & \multicolumn{2}{|c|}{ Male } & \multicolumn{2}{|c|}{ Female } & \\
\hline & & -2005 & $2006^{-}$ & -2005 & $2006^{-}$ & -2005 & $2006^{-}$ & -2005 & $2006^{-}$ & \\
\hline N.Z.* & $2000-2010$ & 1.27 & 1.82 & 0.89 & 2.04 & 21.55 & 22.30 & 9.37 & 10.45 & 8.71 \\
\hline Estonia & $1997-2012$ & 4.93 & 1.74 & 0.97 & 0.46 & 23.96 & 15.09 & 5.86 & 5.15 & 7.27 \\
\hline Finland & 1996-2012 & 1.44 & 0.98 & 1.01 & 0.37 & 19.59 & 18.05 & 5.97 & 6.46 & 6.73 \\
\hline Canada & $2000-2011$ & 1.78 & 1.48 & 1.48 & 1.44 & 14.59 & 11.85 & 5.26 & 5.03 & 5.36 \\
\hline Chile & 1997-2011 & 1.61 & 2.23 & 0.80 & 1.56 & 10.61 & 15.87 & 3.91 & 6.32 & 5.36 \\
\hline Norway & 1996-2012 & 2.06 & 0.89 & 0.77 & 0.65 & 15.25 & 10.94 & 6.82 & 5.17 & 5.32 \\
\hline Slovenia & $1997-2010$ & 3.00 & 0.83 & 1.20 & 0.00 & 14.53 & 11.96 & 6.14 & 4.29 & 5.24 \\
\hline Poland & 1999-2012 & 2.02 & 1.75 & 0.51 & 0.80 & 15.09 & 15.07 & 2.58 & 2.84 & 5.08 \\
\hline Korea & 1995-2012 & 1.23 & 1.52 & 1.52 & 1.80 & 8.51 & 9.11 & 6.42 & 7.70 & 4.73 \\
\hline Austria & $2002-2012$ & 1.00 & 1.00 & 0.32 & 0.65 & 15.53 & 10.39 & 4.88 & 2.67 & 4.56 \\
\hline Australia & $1998-2011$ & 0.96 & 0.84 & 0.54 & 0.59 & 13.19 & 10.94 & 4.63 & 4.17 & 4.48 \\
\hline Belgium & $1998-2010$ & 1.58 & 1.22 & 0.92 & 1.00 & 12.62 & 9.18 & 3.91 & 4.53 & 4.37 \\
\hline U.S. $^{\dagger}$ & 1999-2010 & 1.87 & 1.46 & 0.64 & 0.71 & 12.31 & 11.31 & 2.79 & 2.89 & 4.25 \\
\hline Japan & 1995-2011 & 1.15 & 1.40 & 0.67 & 0.67 & 8.33 & 9.75 & 4.37 & 5.67 & 4.00 \\
\hline Sweden & $1997-2012$ & 0.86 & 0.80 & 0.81 & 1.08 & 7.45 & 9.63 & 4.62 & 5.60 & 3.86 \\
\hline Hungary & 1996-2012 & 1.73 & 0.97 & 0.43 & 0.52 & 10.93 & 9.68 & 3.37 & 2.76 & 3.80 \\
\hline Czech Rep. & 1994-2012 & 1.68 & 0.86 & 0.61 & 0.19 & 11.47 & 9.49 & 2.88 & 2.78 & 3.75 \\
\hline Mexico & 1998-2012 & 1.50 & 1.96 & 0.90 & 1.34 & 7.12 & 8.56 & 2.79 & 3.71 & 3.49 \\
\hline Luxembourg & $1998-2012$ & 0.88 & 0.90 & 2.10 & 0.00 & 12.66 & 4.56 & 2.12 & 2.07 & 3.16 \\
\hline Germany & $1998-2012$ & 0.99 & 0.68 & 0.40 & 0.41 & 8.56 & 6.47 & 2.56 & 2.14 & 2.78 \\
\hline France & 2000-2011 & 1.21 & 1.11 & 0.45 & 0.54 & 7.16 & 6.37 & 2.76 & 2.50 & 2.76 \\
\hline Slovakia & $1994-2010$ & 1.35 & 0.90 & 0.36 & 0.46 & 8.72 & 5.77 & 1.70 & 1.49 & 2.59 \\
\hline Israel & 1998-2011 & 0.69 & 0.56 & 0.36 & 0.38 & 9.60 & 5.54 & 1.67 & 1.45 & 2.53 \\
\hline Denmark & $1994-2012$ & 1.02 & 0.56 & 0.10 & 0.33 & 8.37 & 4.98 & 2.28 & 2.05 & 2.46 \\
\hline Netherlands & 1996-2012 & 1.18 & 0.82 & 0.38 & 0.38 & 5.64 & 5.73 & 2.33 & 2.27 & 2.34 \\
\hline U.K. $\ddagger$ & $2001-2010$ & 0.19 & 0.26 & 0.16 & 0.21 & 4.87 & 4.65 & 1.62 & 1.60 & 1.70 \\
\hline Italy & 2003-2011 & 0.41 & 0.33 & 0.29 & 0.14 & 4.05 & 3.15 & 1.00 & 1.06 & 1.30 \\
\hline Spain & 1999-2012 & 0.51 & 0.34 & 0.17 & 0.18 & 3.98 & 2.75 & 1.46 & 0.96 & 1.29 \\
\hline Portugal & 2002-2012 & 0.17 & 0.41 & 0.36 & 0.18 & 3.22 & 2.80 & 1.76 & 1.25 & 1.27 \\
\hline Mean & (SD) & $1.39(0.91)$ & $1.06(0.52)$ & $0.69(0.46)$ & $0.66(0.53)$ & $11.02(5.16)$ & $9.38(4.71)$ & $3.72(2.00)$ & $3.69(2.25)$ & $3.94(1.80$ \\
\hline
\end{tabular}

${ }^{*}$ New Zealand, ${ }^{\dagger}$ the Unites States, $\neq$ United Kingdom 
group in the period prior to and including 2005. After 2005, the male suicide rate in the older group was 8.85 times higher than that in the younger group. The female suicide rate in the older group was 5.39 times greater than it was in the younger group in the first time period and 5.59 times greater in the second time period.

The male suicide rate was higher than the female rate regardless of country or age. In the 10-14-year age group, the average male suicide rate was 2.01 times greater than the average female suicide rate in the period up to 2005. After 2005, this difference between males and females decreased, but the male suicide rate remained higher, at 1.61 times the female rate. In the 15-19-year age group, the male suicide rate in the first time period was 2.96 times greater than the female rate for that age group, whereas in the second time period, the male suicide rate that was 2.37 times greater than the female rate.

\section{Clustering}

The grid for the SOM was set at $5 \times 5$ in the present study, an appropriate size for the cases we analyzed. Based on the results of the SOM, it was necessary to select the appropriate number of types. We examined the results of classification models obtained through k-means cluster analysis. Figure 1 is a scree plot showing the consensus change in the square of the distance between the observed values and the center of each sub-group when increasing the number of classes. According to Figure 1, the within-group sum of squares appears not to be greatly reduced by selecting more than eight classes; hence, we selected the classification model that included eight types.

Figure 2 shows the results of the SOM, which is expressed in the $5 \times 5$ grid. Relevant cases are presented in this grid, and

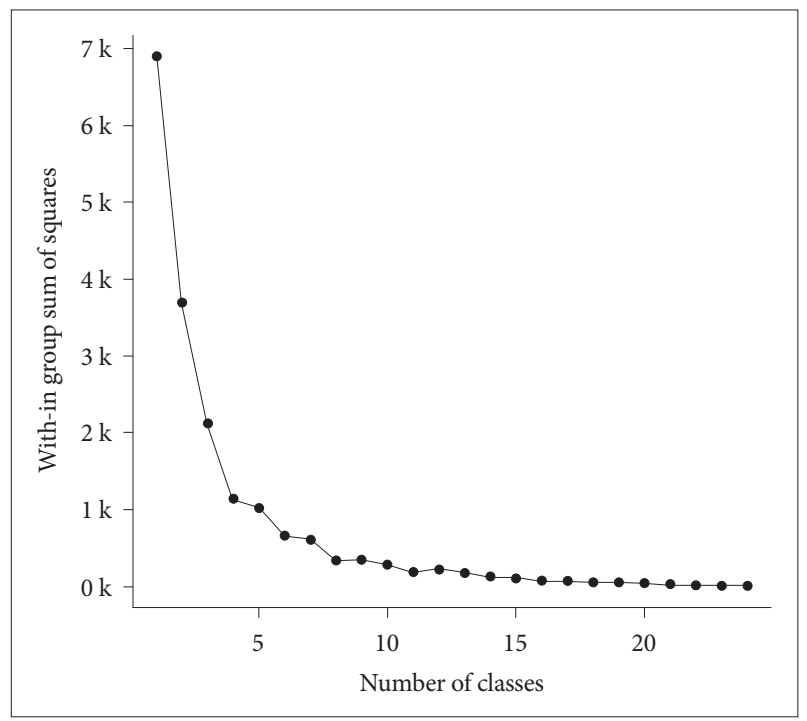

Figure 1. Scree plot for model selection. each is represented as one circle. The two time periods are indicated for each country as 1 (up to and including 2005) and 2 (2006 and later). Cases that are surrounded by the thick lines that divide the grid area into eight regions belong to the same

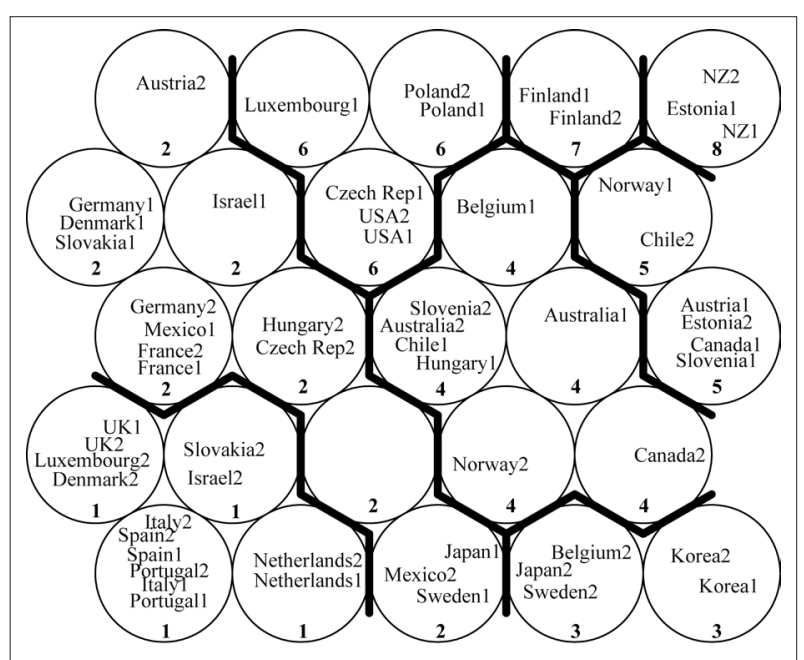

Figure 2. Results of the SOM and classification analyses. NZ: New Zealand, UK: United Kingdom, USA: United States of America.

Table 2. Comparison of types

\begin{tabular}{|c|c|c|c|c|c|}
\hline \multirow{3}{*}{ Type } & \multicolumn{5}{|c|}{ Suicide rates } \\
\hline & \multicolumn{2}{|c|}{ Male } & \multicolumn{2}{|c|}{ Female } & \multirow{2}{*}{ Cases } \\
\hline & $10-14$ & $15-19$ & $10-14$ & $15-19$ & \\
\hline 1 & $\begin{array}{c}-- \\
(0.54)\end{array}$ & $\begin{array}{c}--- \\
(4.41)\end{array}$ & $\begin{array}{c}--- \\
(0.26)\end{array}$ & $\begin{array}{c}--- \\
(1.6)\end{array}$ & 14 \\
\hline 2 & $\begin{array}{c}-- \\
(1.1)\end{array}$ & $\begin{array}{c}-- \\
(8.3)\end{array}$ & $\begin{array}{c}-- \\
(0.55)\end{array}$ & $\begin{array}{c}--- \\
(2.81)\end{array}$ & 14 \\
\hline 3 & $\begin{array}{c}- \\
(1.23)\end{array}$ & $\begin{array}{c}-- \\
(9.24)\end{array}$ & $\begin{array}{c}+++ \\
(1.21)\end{array}$ & $\begin{array}{c}++ \\
(5.98)\end{array}$ & 5 \\
\hline 4 & $\begin{array}{c}- \\
(1.24)\end{array}$ & $\begin{array}{c}= \\
(11.63)\end{array}$ & $\begin{array}{c}- \\
(0.67)\end{array}$ & $\begin{array}{c}= \\
(4.31)\end{array}$ & 8 \\
\hline 5 & $\begin{array}{c}++ \\
(1.97)\end{array}$ & $\begin{array}{c}+ \\
(15.14)\end{array}$ & $\begin{array}{c}+ \\
(0.96)\end{array}$ & $\begin{array}{c}++ \\
(5.76)\end{array}$ & 6 \\
\hline 6 & $\begin{array}{c}+ \\
(1.61)\end{array}$ & $\begin{array}{c}= \\
(12.98)\end{array}$ & $\begin{array}{c}= \\
(0.89)\end{array}$ & $\begin{array}{c}--- \\
(2.68)\end{array}$ & 6 \\
\hline 7 & $\begin{array}{c}- \\
(1.21)\end{array}$ & $\begin{array}{c}+++ \\
(18.82)\end{array}$ & $\begin{array}{c}- \\
(0.69)\end{array}$ & $\begin{array}{c}++ \\
(6.21)\end{array}$ & 2 \\
\hline 8 & $\begin{array}{c}+++ \\
(2.67)\end{array}$ & $\begin{array}{c}+++ \\
(22.6)\end{array}$ & $\begin{array}{l}+++ \\
(1.3)\end{array}$ & $\begin{array}{c}+++ \\
(8.56)\end{array}$ & 3 \\
\hline
\end{tabular}

(a) three minus signs (---) indicate that the suicide rate of this class is less than the $60 \%$ of the overall average mean, (b) two minus signs (--) indicate a suicide rate between $60 \%$ and $80 \%$ of the average mean, (c) a single minus sign (-) indicates a suicide rate between $80 \%$ and $90 \%$ of the average mean), (d) an equal sign (=) indicates a suicide rate between $90 \%$ and $110 \%$ of the average mean, (e) a single plus sign $(+)$ indicates a suicide rate between $110 \%$ and $120 \%$ of the average mean, (f) two plus signs (++) indicate a suicide rate between $120 \%$ and $140 \%$ of the average mean, (g) three plus signs $(+++)$ indicate a suicide rate greater than $140 \%$ of the average mean 
class. Class numbers are presented at the bottom of each grid.

Table 2 presents a comparison of the eight classes with respect to the mean values for suicide rates. For ease of comparison, the suicide rate of each class is indicated using minus (-) or plus $(+)$ signs, depending on its relationship to the average of all classes. The last column displays the class numbers previously referred to in the SOM grid. Types 1 and 2 include the largest number of cases, 14 each, and exhibit suicide rates that are lower than the overall average, with Type 1 exhibiting the lowest suicide rates across categories. In contrast, Type 8 , which includes 3 cases, exhibits the highest suicide rates across the four categories. Many types exhibit similar patterns of suicide rates for the male and female groups, with types that have a relatively low level of male suicide rates also exhibiting relatively low levels of female suicide rates. However, Type 3 proved to be exceptional; this type included both periods for South Korea and the second period for Japan, Sweden, and Belgium; in these cases, the overall suicide rate far exceeded the average despite the fact that male suicide rates were lower than average.

Figure 3 shows the results of the MDS, which was conducted to determine the major dimensions underlying the classification into eight types. We found two major dimensions, which are expressed on the $\mathrm{X}$-axis and $\mathrm{Y}$-axis, and explain $99.6 \%$ of the total variance among the eight types. The sizes of the circles allow for a visual comparison of the four categories of suicide rates; these can then be related to the classification types. The first dimension (Dim 1) underlying differences across classification types appears to represent the overall level of suicide rates. The line plot at the top of Figure 3 shows the differences in mean total suicide rates across types; the

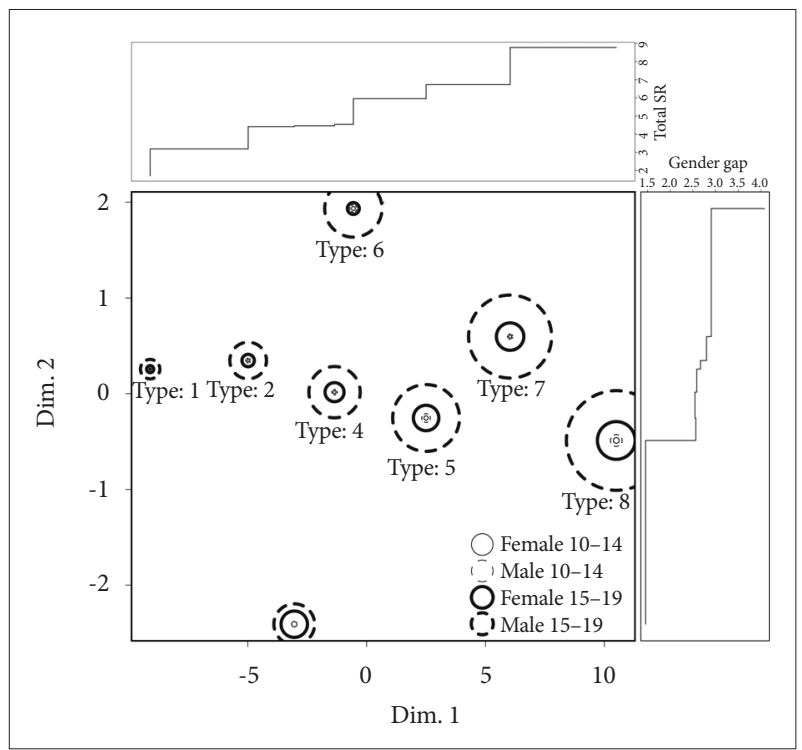

Figure 3. Result of the MDS indicating two major dimensions. Total SR: total suicide rates. mean total suicide rate increases steadily from left to right, reflecting this first dimension. The second dimension of the MDS plot (Dim 2) appears to divide the types according to the gender gap in suicide rates. The line plot on the right side of Figure 3 shows differences in the mean gender gap across types, as represented by a ratio of the male suicide rate to the female rate. The mean gender gap increases by type along this second dimensionon. Most of the types show small degrees of variation with respect to the second dimension, with six types exhibiting similar values on the Y-axis; however, Types 3 and 6 are notable exceptions. These results indicate that most countries have similar gender gaps in their suicide rates among children and adolescence. However, significant outliers exist, exhibiting either a smaller gender gap (i.e., relatively high female suicide rates), as in Type 3 , or a larger gender gap (i.e., relatively high male suicide rates), as in Type 6 .

\section{DISCUSSION}

This study is the latest study comparing the suicide rates of adolescents in OECD countries. We identified significant differences in suicide rates depending on age, sex, country, and time period. Our SOM and MDS analyses revealed that variance in the suicide gender gap is one of the significant dimensions underlying differences in the patterns of child and adolescent suicides.

We found that late adolescence and male gender were universal risk factors for adolescent suicide. Male adolescent suicide rates were about 2-3 times higher than female rates in every country in this study and were higher in late adolescence and in the time period prior to and including 2005. Suicide rates in the 15-19-year age group were dramatically higher than those in the 10-14-year group for most countries, and the effect of age was more prominent among males than females. These results are consistent with previous research. $8,13,15,26,31$

Many countries exhibited a decreasing trend in suicide rates among 10-19-year-olds over time. Several European countries have shown remarkable decreases in male suicide rates, whereas female rates have been stable or have increased. Several studies have offered explanations for this trend, such as restrictions on firearms and some degree of improvement in global health. ${ }^{8,26,35,36}$ It is noteworthy that suicide among female adolescents has not exhibited a similar decline; hence, additional efforts are needed to address female suicide rates. Estonia has exhibited a steep decline in suicide rates in both age groups since 2006, although it still has the second highest suicide rate among the countries studied, with a particularly high incidence among male adolescents. ${ }^{8}$ Although the reason for the recent decrease in adolescent suicides is unclear, the 
country's relatively high suicide rate may be explained by the fact that ethnic Russians have become a non-privileged minority in Estonia since national independence was achieved. ${ }^{37}$

In contrast, Korea and Japan exhibit increasing suicide rates for both genders. Economic recession, inequality, and rapid changes in family structure may be contributing factors to this trend. ${ }^{28,38}$ Korea and Japan have both experienced rapid social and economic changes, and many young people face increasing competitive pressures within an unstable economy and with reduced support due to the weakening of family and community ties. In South Korea, the use of lethal methods of suicide, such as jumping, by both boys and girls, is reported to be primarily responsible for this trend. ${ }^{39}$ Japan's child and adolescent suicide rate may be partially explained by school pressures, weak family support systems, and sexual identity problems at the individual level, and by the economic recession more generally. ${ }^{40,41}$

New Zealand and Finland have the first- and third-highest adolescent suicide rates, respectively, among the 29 OECD countries, and their high rates of adolescent suicide rates have remained steady. These results may reflect cultural backgrounds and societal environments. In the case of New Zealand, where suicide is predominant among indigenous populations, particularly among young boys of Maori ethnicity, the issue of national identity must be considered. ${ }^{727,42}$ Finland has the highest youth suicide rate among European countries. ${ }^{43}$ Some research studies have noted that the high incidence of male adolescent suicide may be due to the frequent use of violent methods, such as firearms. ${ }^{30,43,44}$ In recent years, suicide methods among Finnish female adolescents have become increasingly violent, with a corresponding increase in the female suicide rate. ${ }^{44}$

We extended our research scope using classification methods to compare adolescent suicide rates across OECD countries. Using SOM and MDS analysis, our study classified 58 cases (29 countries over two time periods). Types 1 and 2, which were classified by $\mathrm{k}$-means clustering analysis based on the results of the SOM, had the greatest numbers of member countries, concentrated in southeastern Europe, and were characterized by low suicide rates for both genders. In contrast, Types 7 (Finland) and 8 (New Zealand and Estonia) had high suicide rates for both genders.

The SOM analysis revealed that Types 3 and 6 were distinct from the other types with respect to the male/female ratio in suicide rates. Type 3 includes Belgium, Sweden, and Japan after 2005, and Korea during both time periods; within this type, although overall suicide rates were not high, the rates of female adolescent suicide were relatively high. According to a comparative study of adolescent suicide rates in Belgium and the Netherlands, adolescents in Belgium experienced poorer mental health and more alcohol and drug use than their coun- terparts in the Netherlands, with deliberate self-harm and suicidal thoughts being predominant among females in Belgium. ${ }^{45}$ In Sweden, the rate of female adolescent suicide has increased over the study period. This trend is consistent with a worldwide comparative study of adolescent suicide. ${ }^{26}$ Korea and Japan are the only Asian countries that are members of the OECD; therefore, our results do not fully reflect the Asian situation. However, the culture of male dominance and the low social position of females in Asian culture may play a role in the relatively high incidence of female adolescent suicide in Korea and Japan.

Type 6 countries (Poland, the USA) showed clear gender and age effects. Suicide rates have increased among both boys and girls aged 14 and younger, whereas they have decreased for both genders among adolescents 15 and older. The unique property of these countries is that the male suicide rate is much higher than the female rate. A study of suicide rates in Poland reported that undercounting deaths were especially prominent among females, which may have led to an underestimate in the rate of female suicide. ${ }^{5,46}$ Aggressive suicide methods such as firearm use among males may be related to the high male suicide rate in the USA, but this question needs further investigation.

Male suicide rates are consistently higher than female suicide rates in most countries. The social construction of gender roles is a common explanation for the higher incidence of male suicides. Male gender roles tend to emphasize strength, independence, and risk-taking behavior, and they are likely to prevent males from seeking help for depression and lifethreatening events related to suicidal behaviors. ${ }^{6}$ Furthermore, gender differences in suicide methods are often deemed relevant to the gender gap in suicide rates. Whereas females are more likely to use less immediately lethal methods, males more frequently complete suicide through methods such as hanging, carbon-monoxide poisoning, and the use of firearms. ${ }^{10}$

Despite the general pattern of higher suicide rates among males relative to females, our SOM and MDS analyses revealed that variance in the suicide gender gap is one of the significant dimensions underlying differences in the patterns of child and adolescent suicides. This suggests that cultural and social contexts affecting the gender gap in suicide rates may vary depending on the region and time period. Policies for the prevention of adolescent suicides should be sensitive to such social and cultural contexts.

Methodologically, this research represents the first comparative study of suicide using SOM. Analysis by means of SOM in combination with methods such as k-means clustering and MDS is effective in revealing hidden patterns in high-dimensional data structures such as mortality data across age and 
gender groups. If a suicide data set includes sufficient contextual variables, analysis by SOM may enhance our understanding of the deep structures that describe patterns of child and adolescent suicides.

There are several limitations to this study. First, the study examined 29 OECD countries, consisting largely of European countries with few Asian or American countries. Hence, our results cannot be taken as fully reflecting global trends in adolescent suicide, but rather, as a reflection of trends present in many developed countries; our results do not warrant generalization to developing or under-developed regions. Second, it is possible that our data set underestimated or misclassified a number of suicide events. It is still the case that people tend to underreport suicide events, given that suicide is viewed negatively or is stigmatized in many societies. The reliability of suicide rates may be a significant limitation in comparative suicide studies such as ours. Third, our data set did not include various individual, social, or cultural risk factors that may be related to adolescent suicide rates.

Despite these limitations, our hope is that our study will stimulate additional comparative research regarding rates of child and adolescent suicide and more extensive investigations of the broader psychological, social, contextual, and policy-related variables that may contribute to inter-country variability in adolescent suicide rates.

\section{Acknowledgements}

This study was funded by the Hallym University Research Fund (2014).

\section{REFERENCES}

1. WHO. Preventing Suicide: A Global Imperative. Luxembourg: World Health Organization; 2014.

2. Wasserman D, Cheng Q, Jiang GX. Global suicide rates among young people aged 15-19. World Psychiatry 2005;4:114-120.

3. Wierzbicka A. A Conceptual basis for cultural psychology. Ethos 1993; 21:205-231.

4. Canetto SS, Sakinofsky I. The gender paradox in suicide. Suicide Life Threat Behav 1998;28:1-23.

5. Gmitrowicz A, Szymczak W, Kropiwnicki P, Rabe-Jabłońska J. Gender influence in suicidal behaviour of Polish adolescents. Eur Child Adolesc Psychiatry 2003;12:205-213.

6. Moller-Leimkuhler AM. The gender gap in suicide and premature death or: why are men so vulnerable? Eur Arch Psychiatry Clin Neurosci 2003;253:1-8.

7. Beautrais AL, Fergusson DM. Indigenous suicide in New Zealand. Arch Suicide Res 2006;10:159-168.

8. Värnik A, Kõlves K, Allik J, Arensman E, Aromaa E, van Audenhove $\mathrm{C}$, et al. Gender issues in suicide rates, trends and methods among youths aged 15-24 in 15 European countries. J Affect Disord 2009; 113:216-226.

9. Kim SY, Kim MH, Kawachi I, Cho Y. Comparative epidemiology of suicide in South Korea and Japan: effects of age, gender and suicide methods. Crisis 2011;32:5-14.

10. Schrijvers DL, Bollen J, Sabbe BG. The gender paradox in suicidal behavior and its impact on the suicidal process. J Affect Disord 2012;138: 19-26.
11. McKay K, Milner A, Maple M. Women and suicide: beyond the gender paradox. Int J Culture Ment Health 2014;7:168-178.

12. McMahon EM, Keeley H, Cannon M, Arensman E, Perry IJ, Clarke M, et al. The iceberg of suicide and self-harm in Irish adolescents: a population-based study. Soc Psychiatry Psychiatr Epidemiol 2014;49:1929-1935.

13. McLoughlin AB, Gould MS, Malone KM. Global trends in teenage suicide: 2003-2014. QJM 2015;108:765-780.

14. Beautrais AL. Gender issues in youth suicidal behaviour. Emerg Med (Fremantle)2002;14:35-42.

15. Lewinsohn PM, Rohde P, Seeley JR, Baldwin CL. Gender differences in suicide attempts from adolescence to young adulthood. J Am Acad Child Adolesc Psychiatry 2001;40:427-434.

16. Pfeffer CR. Childhood suicidal behavior: a developmental perspective. Psychiatr Clin North Am 1997;20:551-562.

17. Andrus JK, Fleming DW, Heumann MA, Wassell JT, Hopkins DD, Gordon J. Surveillance of attempted suicide among adolescents in Oregon, 1988. Am J Pub Health 1991;81:1067-1069.

18. Velez CN, Cohen P. Suicidal behavior and ideation in a community sample of children: maternal and youth reports. J Am Acad Child Adolesc Psychiatry 1988;27:349-356.

19. Dervic K, Brent DA, Oquendo MA. Completed suicide in childhood. Psychiatr Clin North Am 2008;31:271-291.

20. OECD. OECD - Social Policy Division - Directorate of Employment, Labour and Social Affairs. CO4.1: teenage suicide (15-19 years old)[online]. Available qt: http://www.oecd.org/els/family/48968307.pdf 2011. Accessed April 17, 2015.

21. Aaron R, Joseph A, Abraham S, Muliyil J, George K, Prasad J, et al. Suicides in young people in rural southern India. Lancet 2004;363:11171118.

22. Phillips MR, Li X, Zhang Y. Suicide rates in China, 1995-99. The Lancet 2002;359:835-840.

23. Zhang J. Marriage and suicide among Chinese rural young women. Soc Forces 2010;89:311-326.

24. Kõlves K, De Leo D. Suicide rates in children aged 10-14 years worldwide: changes in the past two decades. Br J Psychiatry 2014;205:283-285.

25. Rutz EM, Wasserman D. Trends in adolescent suicide mortality in the WHO European Region. Eur Child Adolesc Psychiatry 2004;13:321-331.

26. Kolves K, De Leo D. Adolescent suicide rates between 1990 and 2009: analysis of age group 15-19 years worldwide. J Adolesc Health 2016;58: 69-77.

27. Beautrais AL. Child and young adolescent suicide in New Zealand. Aust N Z J Psychiatry 2001;35:647-653.

28. Cuadrado C, Zitko P, Covarrubias T, Hernandez D, Sade C, Klein C, et al. Association between adolescent suicide and sociodemographic factors in Chile: cross-sectional ecological study. Crisis 2015;36:281-290.

29. Kang EH, Hyun MK, Choi SM, Kim JM, Kim GM, Woo JM. Twelvemonth prevalence and predictors of self-reported suicidal ideation and suicide attempt among Korean adolescents in a web-based nationwide survey. Aust N Z J Psychiatry 2015;49:47-53.

30. Lahti A, Keränen S, Hakko H, Riala K, Räsänen P. Northern excess in adolescent male firearm suicides: a register-based regional study from Finland, 1972-2009. Eur Child Adolesc Psychiatry 2014;23:45-52.

31. McKeown RE, Cuffe SP, Schulz RM. US suicide rates by age group, 19702002: an examination of recent trends. Am J Public Health 2006;96: 1744-1751.

32. Glorioso V, Pisati M. Socioeconomic inequality in health-related behaviors: a lifestyle approach. Qual Quant 2014;48:2859-2879.

33. Lucchini M, Assi J. Mapping patterns of multiple deprivation and wellbeing using self-organizing maps: an application to Swiss household panel data. Soc Indic Res 2012;112:129-149.

34. Kohonen T. Self-organized formation of topologically correct feature maps. Biol Cybernet 1982;43:59-69.

35. Matsubayashi T, Ueda M. The effect of national suicide prevention programs on suicide rates in 21 OECD nations. Soc Sci Med 2011;73:13951400 . 
36. Puzo Q, Qin P, Mehlum L. Long-term trends of suicide by choice of method in Norway: a joinpoint regression analysis of data from 1969 to 2012. BMC Public Health 2016;16:255.

37. Värnik A, Kõlves K, Wasserman D. Suicide among Russians in Estonia: database study before and after independence. BMJ 2005;330:176177.

38. Otzen T, Sanhueza A, Manterola C, Escamilla-Cejudo JA. [Trends in suicide mortality in Chile from 1998 to 2011]. Rev Med Chil 2014;142: 305-313.

39. Park S, Cho SC, Kim BN, Kim JW, Yoo HJ, Hong JP. Increased use of lethal methods and annual increase of suicide rates in Korean adolescents: comparison with adolescents in the United States. J Child Psychol Psychiatry 2014;55:258-263.

40. Koo J, Cox WM. An economic interpretation of suicide cycles in Japan. Contemporary Econ Pol 2008;26:162-174.

41. Hidaka Y, Operario D, Takenaka M, Omori S, Ichikawa S, Shirasaka T. Attempted suicide and associated risk factors among youth in urban Japan. Soc Psychiatry Psychiatr Epidemiol 2008;43:752-757.
42. Fleming TM, Merry SN, Robinson EM, Denny SJ, Watson PD. Self-reported suicide attempts and associated risk and protective factors among secondary school students in New Zealand. Aust N Z J Psychiatry 2007;41:213-221.

43. Lahti A. Epidemiological Study on Trends and Characteristics of Suicide among Children and Adolescents in Finland. Helnsiki: Department of Psychiatry, Helsinki University; 2014.

44. Lahti A, Harju A, Hakko H, Riala K, Rasanen P. Suicide in children and young adolescents: a 25-year database on suicides from Northern Finland. J Psychiatr Res 2014;58:123-128.

45. Portzky G, De Wilde EJ, Van Heeringen K. Deliberate self-harm in young people: differences in prevalence and risk factors between The Netherlands and Belgium. Eur Child Adolesc Psychiatry 2008;17:179186.

46. Höfer P, Rockett IR, Värnik P, Etzersdorfer E, Kapusta ND. Forty years of increasing suicide mortality in Poland: undercounting amidst a hanging epidemic? BMC Public Health 2012;12:644. 